\title{
The structure of Positive Reciprocal Matrices
}

\author{
Shubo Xu \\ Joseph M. Katz Graduate School of Business \\ University of Pittsburgh \\ Pittsburgh, PA 15260
}

\section{Abstract}

In this paper we study the mathematical properties of the set of positive reciprocal matrices with respect the operation Hadamard product. We show that a necessary and sufficient condition for the left and right eigenvectors of a positive reciprocal matrix to be reciprocal of each other is that the row sum coincides with the column sum of the of the standard form of the matrix.

\section{Introduction}

In priority setting with the Analytic Hierarchy Process, it is important to use the two sides of human experience (to dominate and to be dominated, or larger than and smaller than) to obtain a "balanced or reasonable" priority. The main issue here is: what is the relationship between left and right principal eigenvectors of a positive reciprocal matrix?

Let $A=\left(a_{i j}\right)$ be a positive reciprocal matrix and let $w=$ $\left(w_{1}, w_{2}, \ldots, w_{n}\right)$ and $v=\left(v_{1}, v_{2}, \ldots, v_{n}\right)$ be the principal right and left eigenvectors of $A$, respectively. A necessary and sufficient condition for $w$ and $v$ to be reciprocal, $i . e ., w_{i} v_{i}=1$, for all $i$, is given by (Xu [2]):

$$
\mathrm{Ee}=\mathrm{E}^{\top} \mathrm{e}
$$

where $E=\left(e_{i j}\right), e_{i j}=a_{i j} w_{j} / w_{i}$, and $e=(1,1,1, \ldots, 1)^{\top}$. This condition provides additional information on the mathematical structure of $A$. In section 2 we introduce the topological group of positive reciprocal matrices, and in section 3, we extend these results to study the reciprocity relationship between components of the principal left and right eigenvectors.

\section{The Topological Group of Positive Reciprocal Matrices}

Let $\mathbb{N}$ be the set of $n \times n$ positive reciprocal matrices,

$$
\mathbb{N}=\left\{A=\left(a_{i j}\right)_{n \times n} \mid a_{i j} \in \mathbb{R}^{+}, a_{j i}=1 / a_{i j}, \forall i j\right\}
$$

where $\mathbb{R}^{+}$is the set of positive real numbers, and let $\mathbb{C}$ be the set 
of consistent positive reciprocal matrices,

$$
\mathbb{C}=\left\{A=\left(a_{i j}\right)_{n \times n} \mid a_{i j}=a_{i k} a_{k j}, \forall i, j, k\right\} \subset \mathbb{N}^{+}
$$

The Hadamard Product $C=A \circ B=\left(c_{i j}\right)$ of two matrices $A=\left(a_{i j}\right)$; and $B=\left(b_{i j}\right)$ of the same dimension is given by:

$$
c_{i j}=a_{i j} b_{i j}, \quad \forall i, j
$$

Let II denote the set of standard forms of nxn positive reciprocal matrices, i.e.,

$$
I=\left\{E=\left(e_{i j}\right), E \in \mathbb{N}, \sum_{j} e_{i j}=\sum_{j} e_{k j}, \forall i, k\right\}
$$

It is clear that for every $A \in \mathbb{N}$ there exists an unique standard form $E=\left(e_{i j}\right)$ such that $A=E \circ W$. Note that

$$
E=A \circ W^{\top} \text {. }
$$

Saaty [1] calls the matrix defined by ( 6 ) the perturbation matrix.

Now we give without proof because of their simplicity some properties that the set of reciprocal matrices $\mathbb{N}$ satisfy:

(1) $\mathbb{N}^{+}$is a commutative group under the Hadamard product.

(2) $\mathbb{C}$ is a normal subgroup of $\mathbb{N}$

(3) Let $\mathscr{F}$ be a direct product of the sets $\mathbb{C}$ and $\mathbb{I}$, i.e. $\mathscr{F}=\left\{A=\left(a_{i j}\right) \mid A=C \circ E, C \in \mathbb{C}, E \in \mathbb{I}\right\}$. We have $\mathscr{F}=\mathbb{N}$.

Let $H$ be a decomposition of $\mathbb{N}$ into classes such that to every reciprocal matrix $A$ there corresponds a matrix $E \in \mathbb{I}$ and a matrix $M_{c} \in \mathbb{C}$ such that $A=E \circ M$. Obviously, these subclasses are disjoined.

(4) $\mathcal{H}$ is a regular decomposition of $\mathbb{N}$.

Let $\mathbb{N}_{E}$ be the set of all the subclasses mentioned above, i.e. it consists of all of Hadamard products of each $E \in \mathbb{I}$ and every $M_{c} \in \mathbb{N}$.

(5) $\mathbb{N}_{E}$ is a commutative group under the Hadamard product.

(6) $\mathbb{N}_{E}$ is a quotient group of $\mathbb{N}$ under the regular decomposition $\mathrm{H}$, i.e., $\mathbb{N}_{\mathrm{E}}=\mathbb{N} / \mathbb{C}$.

(7) $\mathbb{N}$ is homomorphic to $\mathbb{N}_{E}$.

(8) There is a 1-1 correspondence between the quotient group $\mathbb{N} / \mathbb{C}$ and $\mathbb{I}$.

(9) $\mathbb{C}$ is a kernel of the homomorphism that maps $\mathbb{N}$ into $\mathbb{N}_{\varepsilon}$.

(10) $\mathbb{N}$ is a differentiable topological group homomorphic to $\mathbb{R}_{n(n-1) / 2}^{+}$.

(11) $\mathbb{C}$ is a differentiable topological group homomorphic to $\mathbb{R}_{n-1}^{+}$. 
3. An Application of the Topological Group of Positive Reciprocal Matrices

In priority setting it.is necessary to use the two sides of human experience to obtain a "balanced or reasonable" priority. Mathematically, the problem can be considered as a question of how to develop the matrix of dominance or the matrix of domination, or perhaps how to synthesize the left and right eigenvectors of the pairwise comparison matrix. It is easy to show that the components of the principal left and right eigenvectors of consistent matrices are reciprocals of each other. However, when the positive reciprocal matrices are inconsistent, the above reciprocal property does not obtain. The general condition for which the reciprocal property holds is important to synthesize the left and right eigenvectors of the positive reciprocal matrices.

Theorem 1. The reciprocal property between corresponding components of the left and right principal eigenvectors of a positive reciprocal matrix A holds is and only if the standard form of the matrix $A$ and the transpose of the standard form have the same row sums.

Proof: Let $E$ be the standard form of a positive reciprocal matrix $A$, i.e., $E \in \mathbb{I}$, and $A \in \mathbb{N}$. Since $E=A \circ W$, where $W=\left(W_{i} / W_{j}\right)$, and $w=\left(w_{1}, w_{2}, \ldots, w_{n}\right)$ is the principal right principal eigenvector of $A$ corresponding to the principal eigenvalue $\lambda_{\max }$. If $E e=E_{T} e$, we have $E e=\lambda_{\max } e$ and $(E \circ W) w=\lambda_{\max } w$. Let $v=\left(v_{1}, \dot{v}_{2}, \ldots, v_{n}\right)^{r}$ be the left principal vector of $A$, then

$$
\mathrm{A}^{\top} \mathrm{V}=\lambda_{\max } \mathrm{V}
$$

i.e.

since

$$
\left(E^{\top} \circ W^{\top}\right) V=\lambda_{\max } V
$$

$$
\dot{A}^{\top}=\left(E^{\top} \circ W^{\top}\right)=W^{-1} E W
$$

we have

$$
W^{-1} E^{T} W V=\lambda_{\text {max }} V
$$

or 


$$
E^{\top} W v=\lambda_{\max } W v
$$

This shows that $W v$ is the principal right eigenvector of $E$, or the left principal eigenvector of $\mathrm{E}$. Since $\mathrm{E} e=\mathrm{E}^{\top}=\lambda_{\max } \mathrm{e}$, and the principal eigenvector is unique to within a multiplicative constant, we conclude that

$$
\text { Wv }=e
$$

i.e. the corresponding components of the principal left and right eigenvectors are reciprocal.

Conversely, if the reciprocal property (12) holds, and since (7), (10) and (11),

$$
\begin{aligned}
& \text { we have } \\
& E^{\mathrm{T}} e=\lambda_{\max } \mathrm{e}
\end{aligned}
$$

But

therefore

$$
\mathrm{Ee}=\lambda_{\max } \mathrm{e}
$$

$$
\mathrm{Ee}=\mathrm{E}^{\top} \mathrm{e}=\lambda_{\max } \mathrm{e}
$$

This concludes the proof of the theorem.

Corollary. If $A \in \mathbb{C}$, the reciprocal property between corresponding components of the principal left and right eigenvectors holds.

For any $3 \times 3$ positive reciprocal matrix, it is easy to verify that the row sum of its standard form is equal to the row sum of the standard form of its transpose. So that, we have the following corollary

Corollary. For any $3 \times 3$ positive reciprocal matrix, the reciprocal property between the principal right and left eigenvector holds.

There exists a special kind of positive reciprocal matrices for which the reciprocal property holds. To introduce this group of positive reciprocal matrices we need the following definition.

Definition. A set $\Omega$ is called the inverse invariant set if

$$
F(\Omega)=\Omega
$$

where

$$
F: \mathbb{R}-\mathbb{R}, F(\Omega)=\{1 / s \mid s \in \Omega\}
$$




$$
A=\left[\begin{array}{lllll}
1 & 2 & 1 / 16 & 8 & 1 / 2 \\
1 / 2 & 1 & 1 / 2 & 1 & 16 \\
16 & 1 / 2 & 1 & 32 & 2 \\
1 / 8 & 1 & 1 / 32 & 1 & 4 \\
2 & 1 / 16 & 1 / 2 & 1 / 4 & 1
\end{array}\right]
$$

Its normalized right eigenvector is given by:

$$
\mathrm{W}=(0.1250,0.2500,0.5000,0.0625,0.0625)^{\mathrm{T}}
$$

and $\lambda_{\max }=9.5$, C.I. $=1.12>0.1$. This matrix is very inconsistent. The perturbed matrix is given by:

$$
\mathrm{E}=\left[\begin{array}{llllll}
1 & 4 & 1 / 4 & 4 & 1 / 4 \\
1 / 4 & 1 & 4 & 1 / 4 & 4 \\
4 & 1 / 4 & 1 & 4 & 1 / 4 \\
1 / 4 & 4 & 1 / 4 & 1 & 4 \\
4 & 1 / 4 & 4 & 1 / 4 & 1
\end{array}\right]
$$

Note that the sets $S_{1}, S_{2}, S_{3}, S_{4}, S_{5}$, which consist of the elements in each of the rows of the perturbed matrix, are inverse-invariant sets. The left eigenvector of $A$ corresponding to $\lambda_{\max }=9.5$ is given by:

$$
v=(0.1739,0.0870,0.0435,0.3478,0.3478)^{\top}
$$

The corresponding components of the left and right principal eigenvectors $\mathrm{v}$ and $\mathrm{w}$ are reciprocal with the multiplicative constant 46 , i.e., $v^{\top} w=46$. If the decision maker still wants to set up a priority for the above matrix $A$, it is suggested the selection of the balance priority vector given by:

$$
p=1 / 2(w+v)=(0.1495,0.1685,0.2718,0.2051,0.2051)_{T}
$$

The above example indicates that the reciprocal property of the components of the left and right eigenvectors of a positive reciprocal matrix can also hold even though the index of consistency of the matrix is high. The key point to determine the existence of the reciprocal property for a positive reciprocal matrix $A$ is not the index of consistency of the matrix $A$ but the difference between the sums of the rows and the columns of the perturbed matrix $\mathrm{E}$ of the matrix $\mathrm{A}$. 
5. Conclusion

The topological group theory shown in this paper reveals the basic mathematical structure of positive reciprocal matrices. It is straightforward to extend the theory presented here to judgment matrices, because they are a special kind of positive reciprocal matrices whose elements belong to the set $\{1,2, \ldots, 9,1 / 2, \ldots$, $1 / 9\}$. Another field that concerns topological group theory is the study of the consistency of the judgment process in group decision making.

\section{REFERENCES}

[1] T.I. Saaty, 1980, The Analytic Hierarchy Process, McGraw-Hill Company.

[2] Shubo Xu, 1988, "Characterizing Principal Left-right Eigenvector Reciprocity in a positive Reciprocal Matrix", Proceedings of the International symposium on AHP, Sept. 6-9, 1988, Tianjin, China

[3] D. Montgomery and L. Zippin, 1955, Topological Transformation Group, Interscience Publishers, Inc., New York

[4] D.J.S. Robinson, 1980, A course in the Theory of Group, Springer-Verlag, New YYork 